\title{
What next for Mars?
}

\author{
Future missions to the red planet require coordination — and a keen eye on costs.
}

$\uparrow$ he story of the Phoenix Mars lander (see page 690) raises nagging questions about how to study Earth's most interesting neighbour. Mars has been visited by spacecraft more often than any other planet. Yet the frequency of the probes has made them no cheaper. The Phoenix mission, a bargain by NASA standards, cost more than US $\$ 400$ million - almost half the annual budget of the US Geological Survey that studies Earth's terrain. NASA's next planned mission to the Martian surface, a rover known as the Mars Science Laboratory (MSL), has a budget that was pushing $\$ 2$ billion even before last week's announcement that its launch date was to be delayed by two years; it will now comfortably exceed that sum.

Granted, investment on this scale has produced stunning science. Mars has been mapped from orbit in exquisite detail. The remarkably long-lived Spirit and Opportunity rover missions, which landed five years ago next month, have produced a rich scientific understanding of the surface. And Phoenix itself has revealed a polar environment more dynamic than anything yet seen on the planet, with frosts coming and going, clouds dropping gentle snows and subsurface ice vanishing into vapour.

What this huge investment has not produced, however, is the longterm infrastructure and reusable technology that would make future missions more affordable. To take one example: the most difficult part of any mission to the Martian surface is landing, yet every such mission to date has used technology tailored from scratch. There are currently no plans to reuse the rocket landing system painstakingly developed for Phoenix mission, nor the air bags of the rover missions, nor even the ambitious 'sky crane' system that will supposedly lower MSL to the surface from a kind of rocket-powered hovercraft.

This constant reinvention is an indulgence that planetary exploration programmes can no longer afford. The United States and Europe, which have so far led the way in Mars exploration, should also lead the way in this respect by jointly reusing technology wherever it is remotely feasible. Rather than developing a new parachute-braking system, for example, the ExoMars rover being planned by the European Space Agency (ESA) could commit to using the air-bag technology deployed by NASA's rovers, and use as many of the other components from those missions as possible.

Looking further ahead, NASA, ESA and perhaps the new spacefaring nations in Asia should jointly start developing standard technologies for Mars exploration. One example might be a landing system designed to deliver hardy sensors to the surface at high speed, allowing the piecemeal, pay-as-you-go deployment of a planet-wide network to monitor Mars's meteorology and seismology. Another might be a shared, longlived communications infrastructure: satellites in orbit around Mars with large antennas pointed towards Earth that are designed to relay even weak signals from the various science payloads. Such a system would mean that payloads could be built much more cheaply.

Meanwhile, the most effective way to improve the returns on Mars exploration would be better cost discipline. Missions understandably go over budget. There is always pressure to include more instruments, not least because researchers are aware of how few opportunities there are. And the incentives to be realistic in initial budgeting are relatively small, as the missions are unlikely to be cancelled if the costs escalate.

Cancelling, or radically downscaling, overbudget missions such as MSL would set science back in the short term. It would also be difficult politically, as aerospace contractors and NASA's spacecraftbuilding centres have a lot of clout. But the only way to make Mars exploration a more regular affair is to stop the missions from costing too much. Eventually, a virtuous circle could be established: cheaper missions would mean more of them, which would mean less pressure to overload each one, in turn keeping the costs down. The fact that a series of failures followed an attempt to do this in the 1990s does not mean it was the wrong thing to do; it means that the effort needs clearer management and accountability, technology that can be reused from mission to mission and patience.

The hard truth is that trying to understand one planet from the surface of another is going to be a long, drawn-out affair. The early history of the space age, in which ancient points of light turned overnight into worlds as complex as our own - accompanied by detailed and often spectacular images - promised an unsustainable pace of progress. The progress of planetary science now demands time and a sound strategy.

\section{Watching Big Brother}

The world is sleepwalking into a surveillance society. A European court ruling offers a timely wake-up call.

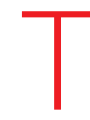

he European Court of Human Rights last week issued an opinion to which the developers and users of new technologies should pay heed. "Any state claiming a pioneer role in the development of new technologies," the court said, bears special responsibility for "carefully balancing the potential benefits of the extensive use of such techniques against important private-life interests."

This timely reminder, coming just before the 60th anniversary on 10 December of the Universal Declaration of Human Rights, was a strike at the British government's policy of keeping DNA fingerprint records on an ever-growing number of innocent people in England and Wales. That policy was indeed pioneering: the United Kingdom authorized the world's first national DNA database in 1994. The database has since proved helpful in solving a small but significant number of crimes, and many countries have followed suit. But the system, originally intended to cover only people convicted of serious 